Contributed Talk, for presentation at the 9 th International Conference on $\mathrm{X}$-ray Absorption Fine Structure (XAFS), to be held in Grenoble, France on 8/25-30/96.

$$
\text { CONF- } 9608104--4 \quad \text { ANL/CHM/CP- }-89746
$$

NANOSCALE ENCAPSULATION: THE STRUCTURE OF CATIONS IN HYDROPHOBIC MICROPOROUS ALUMINOSILICATES

Stephen R. Wasserman*, Steven E. Yuchs, Daniel Giaquinta, L. Soderholm, and Kang Song

Chemistry Division

Argonne National Laboratory

9700 S. Cass Ave.

Argonne, IL 60439

USA

708-252-3527

708-252-9288 (FAX)

Email: SRW@ANL.GOV

Bem C IVE

SEP 031996

OSTI

\begin{abstract}
Hydrophobic microporous aluminosilicates, created by the organic surface modification of inherently hydrophilic materials such as zeolites and clays, are currently being investigated as storage media for hazardous cations. The use of organic monolayers to modify the surface of an aluminosilicate after introduction of an ion into the zeolite or clay reduces the interaction of water with the material. The resultant systems are approximately 20 times more resistant to leaching of stored ion than the unmodified ion-exchanged materials. XAS spectra from the encapsulated ion demonstrate that byproducts from the organic modifier can complex with the stored cation. This complexation can result in a decreased affinity of the cation for the aluminosilicate matrix. Changing the organic modifier eliminates this problem. XAS spectra also indicate that the reactivity and speciation of the encapsulated ion may change upon application of the hydrophobic layer.
\end{abstract}

Short Title: Nanoscale Encapsulation

*Author to whom correspondence should be addressed

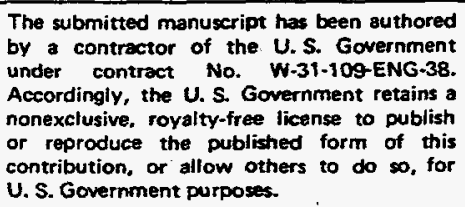




\section{DISCLAIMER}

Portions of this document may be illegible in electronic image products. Images are produced from the best available original document. 


\section{DISCLAIMER}

This report was prepared as an account of work sponsored by an agency of the United States Government. Neither the United States Government nor any agency thereof, nor any of their employees, makes any warranty, express or implied, or assumes any legal liability or responsibility for the accuracy, completeness, or usefulness of any information, apparatus, product, or process disclosed, or represents that its use would not infringe privately owned rights. Reference herein to any specific commercial product, process, or service by trade name, trademark, manufacturer, or otherwise does not necessarily constitute or imply its endorsement, recommendation, or favoring by the United States Government or any agency thereof. The views and opinions of authors expressed herein do not necessarily state or reflect those of the United States Government or any agency thereof. 


\title{
NANOSCALE ENCAPSULATION: THE STRUCTURE OF CATIONS IN HYDROPHOBIC MICROPOROUS ALUMINOSILICATES
}

Stephen R. Wasserman, Steven E. Yuchs, Daniel Giaquinta, L. Soderholm, and Kang Song Chemistry Division, Argonne National Laboratory, 9700 S. Cass Ave., Argonne, Il 60439, USA

\begin{abstract}
Hydrophobic microporous aluminosilicates, created by the organic surface modification of inherently hydrophilic materials such as zeolites and clays, are currently being investigated as storage media for hazardous cations. The use of organic monolayers to modify the surface of an aluminosilicate after introduction of an ion into the zeolite or clay reduces the interaction of water with the material. The resultant systems are approximately 20 times more resistant to leaching of stored ion than the unmodified ion-exchanged materials. XAS spectra from the encapsulated ion demonstrate that byproducts from the organic modifier can complex with the stored cation. This complexation can result in a decreased affinity of the cation for the aluminosilicate matrix. Changing the organic modifier eliminates this problem. XAS spectra also indicate that the reactivity and speciation of the encapsulated ion may change upon application of the hydrophobic layer.
\end{abstract}

\section{Introduction}

The applications of X-ray absorption spectroscopy (XAS) to the study of environmental problems generally fall into two general categories. Investigations in the first group utilize XAS to determine the species present in contaminated samples. In the second type of application, XAS examines storage materials for hazardous waste, in order to investigate the efficacy of such systems in excluding hazardous compounds from the environment.

Microporous aluminosilicates, including clay minerals and zeolites, are ion-exchange materials. [1,2] In their most common forms, they have the ability to incorporate cationic species within their matrices. Because of this property, microporous aluminosilicates have 
been proposed as storage media for hazardous waste. In this paper we use XAS to examine the structure of cations held within smectite clay minerals and to determine how modification of the surface of the clay affects the coordination of the stored cation.

Smectite clays consist of aluminosilicate sheets. ${ }^{1}$ These sheets contain a central section of octahedrally coordinated $\mathrm{Al}^{3+}$ that is sandwiched between two layers of tetrahedrally coordinated $\mathrm{Si}^{4+}$. Random substitutions, generally of $\mathrm{Mg}^{2+}$ for $\mathrm{Al}^{3+}$ and $\mathrm{Al}^{3+}$ for $\mathrm{Si}^{4+}$, result in a net negative charge in the lattice. This charge is balanced by cations in the interlayer between the sheets. The interlayer also contains water, some of which is complexed to the cations. The structure of smectite clays is illustrated in Figure 1. The interlayer cations can be replaced by ion-exchange techniques with other positively charged species. For this study we have replaced the cations originally present in a clay, usually sodium, calcium, and potassium, with cationic metal species, including copper(II), nickel(II), $\mathrm{Pb}(\mathrm{II})$, and uranyl $\left(\mathrm{UO}_{2}{ }^{2+}\right)$. The clay used for these experiments is bentonite, a form of the smectite clay montmorillonite.

Clays are naturally hydrophilic. This affinity for water, however, also facilitates the leaching of any hazardous species stored within the mineral. In order to isolate the cationic species within the clay interlayer, we have modified the surfaces of ion-exchanged smectite clays using alkylsilanes of the form $\mathrm{RSiX}_{3}, \mathrm{R}=\mathrm{C}_{2} \mathrm{H}_{5}-, \mathrm{C}_{18} \mathrm{H}_{37-}, \mathrm{X}=-\mathrm{Cl},-\mathrm{OCH}_{3}$ (Figure 1). The organosilanes bind covalently to the surface of the clay mineral and render the mineral hydrophobic. The presence of this coating inhibits the exchange of external water into and out of the interlayer. The exclusion of water reduces the leaching of ions from the interlayer back into the environment.

\section{Experimental Section}

The general preparation of ion-exchanged clays has been described elsewhere.[3] The $\mathrm{Ca}^{2+}$ form of bentonite was used as received (bentolite L, Southern Clay Products, Gonzales, Texas). The surfaces of the ion-exchanged clays were coated with octadecyltrimethoxysilane, $\mathrm{CH}_{3}\left(\mathrm{CH}_{2}\right)_{17} \mathrm{Si}\left(\mathrm{OCH}_{3}\right)_{3}$ (OTS, Aldrich), and ethyltrichlorosilane, $\mathrm{CH}_{3}\left(\mathrm{CH}_{2}\right)_{17} \mathrm{SiCl}_{3}$ (ETS, 
Aldrich). The silanes were distilled and stored under dry nitrogen prior to use. The clay ( $1 \mathrm{~g})$ and silane $(1 \mathrm{~mL})$ were added to anhydrous hexane $(40 \mathrm{~mL})$. The mixture was stirred for 24 hours at room temperature under a dry nitrogen atmosphere. 3-indolepropionic acid (0.01g) was added as a catalyst for the reaction of the trimethoxy silane with the clay surface. The samples were collected by centrifugation and washed three times with anhydrous hexane (25 $\mathrm{mL}$ ) to remove any unreacted silane. After the final wash, the hydrophobic clay was dried at room temperature. The process for the formation of the surface coatings is based on previous work on self-assembled monolayers. $[4,5]$

Hydrothermal processing of the clay samples was performed in a sealed stainless steel Parr high pressure bomb (4746) with a Teflon® insert that contained clay (250 $\mathrm{mg})$ and deionized water $(10 \mathrm{~mL})$. The bomb was placed into a Lindberg crucible furnace and heated at $1^{\circ}$ per minute to $200^{\circ} \mathrm{C}$. The temperature was maintained at $200{ }^{\circ} \mathrm{C}$ for 20 hours, after which the samples were cooled radiatively to room temperature.

X-ray absorption spectra were obtained at the National Synchrotron Light Source (NSLS) on beamlines X23A2 and X10C, and at the Stanford Synchrotron Radiation Laboratory (SSRL) on station 4-3. The beamlines were equipped with either a $<220>$ (SSRL and X10C) or $<311>(\mathrm{X} 23 \mathrm{~A} 2)$ double-crystal Si monochromator. Harmonics were rejected on $\mathrm{X} 10 \mathrm{C}$ by use of a mirror and at SSRL by detuning the monochromator to approximately $50 \%$ of the maximum X-ray intensity. Data were collected in fluorescence mode using a flow-type fluorescent ion chamber (Lytle) detector (The EXAFS Co.) that was purged with Ar gas. An appropriate 3 absorption lengths filter was placed between the sample and the ion chamber to minimize artifacts caused by scattering of the incident X-ray beam. Calibration of the edges was maintained through simultaneous acquisition of the transmission spectrum of a reference material. The position of $\mathrm{k}=0 \AA^{-1}$ was defined as the maximum in the derivative spectra from the reference. Analysis of the EXAFS data was performed using theoretical phase shifts and scattering amplitudes from FEFF 3.25.[6] 


\section{Results and Discussion}

Figure 2 shows the near edge spectra (XANES) and radial structure functions for copper(II) ions in the interlayers of bentonite (Cu-bentonite). These spectra illustrate the coordination environment of the ion before and after addition of organic monolayers formed from ethyltrichlorosilane (ETS) and octadecyltrimethoxysilane (OTS). The clays that result from addition of these coatings are both hydrophobic. Indeed, they float on water, despite having a density considerably greater than $1 \mathrm{~g} / \mathrm{mL}$. However, the utilization of the two types of silane to create monolayers on the clay surface results in distinct effects on the local coordination of the copper ion. The resolved shoulder at $8988.5 \mathrm{eV}$ in the $\mathrm{Cu}$ edge of the original Cu-bentonite, which corresponds to the $1 \mathrm{~s}-4 \mathrm{p}_{\mathrm{z}}$ transition, is indicative of a copper ion in a square planar environment.[7,8] The EXAFS data indicate that the coordinating species are oxygen atoms, presumably from water molecules contained within the interlayer of the clay. The XANES and radial distributions after addition of OTS are identical to those of the original $\mathrm{Cu}$-bentonite. We therefore conclude that the modification of the clay surface using OTS does not alter the speciation of the copper. In contrast, when ETS is used to form the monolayer, both the near edge absorption and the EXAFS change. The XANES spectrum shifts approximately $1.5 \mathrm{eV}$ to lower energy. At the same time the first coordination sphere in the radial distribution separates into two distinct peaks. The peak at $r^{\prime}=1.55 \AA$ (not-phase corrected) in the radial structure function reflects the continued ligation of the copper by oxygen atoms. That at $r^{\prime}=1.96$ apparently indicates the replacement of approximately half of the coordinating water molecules by chloride ions, $\mathrm{Cl}^{-}$. The EXAFS fitting parameters for these three systems are summarized in Table 1.

This change in the local coordination affects the ability of the clay-monolayer system to retain the interlayer ion. In testing the resistance of these systems to leaching of the interior cation, we find that the hydrophobic clay created using OTS is approximately 20 times more effective than the original $\mathrm{Cu}$-bentonite in retaining the copper ion.[9] The hydrophobic clay that results from the use of ETS as the organic modifier is slightly worse than the original $\mathrm{Cu}$ - 
bentonite in keeping the copper within the aluminosilicate matrix. We believe that this behavior results from the complexation of chloride anion with the copper. The reaction of an alkyltrichlorosilane, $\mathrm{RSiCl}_{3}$, with the clay surface, and the water found there, forms siloxane bonds, -Si-O-Si-. In the process, hydrochloric acid, $\mathrm{HCl}$, is also created. Some of the negative chloride ions from the acid replace neutral water molecules as ligands of the copper ion. Exchange of two or more water molecules by chlorides creates a complex that is no longer positively charged. The coulombic forces which normally hold the cation in place are no longer effective. The positive charge in the interlayer, formerly located on the copper species, is probably now centered on protons $\left(\mathrm{H}^{+}\right)$from the $\mathrm{HCl}$. As a result the chlorinated copper species are easily leached from the mineral. Similar behavior, both in the changes in the XAS spectra and in resistance to leaching, is observed when nickel(II), lead(II), or uranyl $\left(\mathrm{UO}_{2}{ }^{2+}\right)$ cations are used in place of copper in these systems.

Hydrothermal processing. In order to simulate the effects of long term storage on these materials, as well as the effects of extreme conditions which may occur in a geologic repository, we have subjected both hydrophobic and hydrophilic clays that contain uranyl $\left(\mathrm{UO}_{2}{ }^{2+}\right.$ ) cations to hydrothermal processing in water at $200^{\circ} \mathrm{C}$ and $15.8 \mathrm{~kg} / \mathrm{cm}^{2}$ (calc).[10] XAS was used to examine the speciation of the uranium before and after this type of processing.

Figure 3 shows the near edge spectra and radial distributions for uranyl in bentonite before and after hydrothermal treatment. Figure 3 also shows the XANES from a uranylbentonite after both addition of an organic monolayer, formed from octadecyltrimethoxysilane, and exposure to hydrothermal conditions. For the simple uranyl-bentonite, the XANES and radial structures are unchanged after hydrothermal processing. The two peaks in the radial distribution between $r^{\prime}=1$ and $2.5 \AA$ reflect the presence of two axial oxygen atoms, which are directly bound to the uranium, and approximately 5 equatorial oxygen atoms from water molecules which are complexed to the uranyl species. The spectra of a uranyl clay after application of an organic monolayer but prior to hydrothermal treatment (not shown) are 
identical to the uranyl-bentonite shown in Figure 3. However, when the monolayer is present, exposure to hydrothermal conditions results in an apparent change in the oxidation state of the uranium. The position of the absorption edge, as determined from the maximum of the derivative spectrum, shifts approximately $3 \mathrm{eV}$ to lower energy. This shift is confirmed by the lowering of the energy parameter in the EXAFS fits by $3.5 \mathrm{eV}$. At the same time, the EXAFS data confirm that the local coordination of the uranium has altered. The axial and equatorial features of uranyl have disappeared, to be replaced by a single peak centered at $r^{\prime}=1.8 \AA$. Simultaneously, a uranium-uranium correlation appears at $r^{\prime}=4 \AA$. These changes are consistent with the reduction of uranium to the +4 oxidation state, and the aggregation of the uranium into small, disordered $\mathrm{UO}_{2}$ particles. We cannot rule out the possibility that the shoulder in the radial distribution at $r^{\prime}=1.42 \AA$ indicates the presence of a small amount of unmodified uranyl cations. We have observed a similar reduction of $\mathrm{Cu}(\mathrm{II})$ upon the heating of $\mathrm{Cu}$ (II)-bentonite that has been modified by OTS.

\section{Conclusion}

These experiments have demonstrated the utility of XAS in evaluating how surface modification affects the chemical and physical structure of ions held within the interlayers of clay minerals. XAS has also illuminated the reductive processes which can occur within these interlayers when hydrophobic clays are subjected to hydrothermal processing. The latter results can serve as models for the interactions which occur in nature between ions, clay minerals, and organic compounds.

\section{Acknowledgments}

This research was supported by the Strategic Environmental Research and Development Program of the U. S. Department of Defense and the Division of Chemical Sciences, Office of Basic Energy Sciences, U. S. Department of Energy under contract W-31-109-ENG-38. 


\section{Figure Captions}

Figure 1. Structure of smectite clay minerals and schematic representation of ion exchange and organic encapsulation of ions within a smectite.

Figure 2. (A) X-ray absorption near edge spectra (XANES) and (B) radial structure functions (not phase corrected) for copper(II) in the interlayer of the smectite clay mineral, bentonite: (1) ion-exchanged clay $(-),(2)$ after formation of a monolayer from octadecyltrimethoxysilane $\left(\mathrm{C}_{18} \mathrm{H}_{37} \mathrm{Si}\left(\mathrm{OCH}_{3}\right)_{3}, \ldots\right)$, and (3) after formation of a monolayer from ethyltrichlorosilane $\left(\mathrm{CH}_{3} \mathrm{CH}_{2} \mathrm{SiCl}_{3}, \rightarrow-\right.$ ). The range for the forward Fourier transform was $\Delta \mathrm{k}=1.8-13.0 \AA^{-1}$.

Figure 3. (A) X-ray absorption near edge spectra (XANES) and (B) radial structure functions (not phase cbrrected) for uranyl ( $\mathrm{UO}_{2}{ }^{27}$ ) in the interlayer of bentonite: (1) ion-exchanged clay

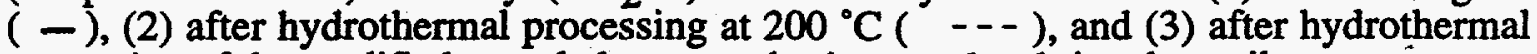
processing of the modified uranyl clay created using octadecyltrimethoxysilane $\left(\mathrm{C}_{18} \mathrm{H}_{37} \mathrm{Si}\left(\mathrm{OCH}_{3}\right)_{3},-\rightarrow\right.$ ). The range for the forward Fourier transform was $\Delta \mathrm{k}=1.0$ $11.0 \AA^{-1}$. 
Table I. Parameters for the First Coordination Shell in the Fluorescence XAS Spectra of Copper(II) in Bentonitea

\begin{tabular}{l||ccccc}
\hline Sample & Element & $\mathrm{n}$ & $\mathrm{r}(\AA)$ & $\sigma^{2} \times 10^{3}$ & $\mathrm{E}$ \\
\hline $\mathrm{Cu}$ - bentonite & 0 & 4.0 & 1.92 & 4.5 & 8.7 \\
& & & & & \\
$\mathrm{Cu}-$ bentonite + & 0 & 4.1 & 1.92 & 4.5 & 8.6 \\
$\mathrm{C}_{18} \mathrm{H}_{37} \mathrm{Si}\left(\mathrm{OCH}_{3}\right)_{3}$ & & & & & \\
$\mathrm{Cu}_{\mathrm{C}-\text { bentonite }+}$ & $\mathrm{O}$ & 2.1 & 1.93 & 6.4 & 8.7 \\
$\mathrm{C}_{2} \mathrm{H}_{5} \mathrm{SiCl}_{3}$ & $\mathrm{Cl}$ & 2.0 & 2.26 & 6.0 & 8.4
\end{tabular}

aThe errors in coordination numbers (n) and radial distance (r) are $\pm 20 \%$ of $n$ and $\pm 0.02 \AA$ respectively. The scale factor was determined assuming $\mathrm{n}=4.0$ for $\mathrm{Cu}$ - bentonite. Windows for Fourier transforms of $\mathrm{k}^{3} \chi(\mathrm{k})$ : forward-1.8 to $13.0 \AA^{-1}$, inverse- 0.7 to $2.35 \AA$. The data were fit in the range from 2.5 to $12.5 \AA^{-1}$. 


\section{References}

[1] Grim R. E., Clay Mineralogy, 2nd ed. (McGraw-Hill, New York, 1968).

[2] Rabo J. A., Zeolite chemistry and catalysis, (American Chemical Society, Washington, D. C., 1976).

[3] Carrado K. A. and Wasserman S. R., J. Am. Chem. Soc. 115 (1993) 3394-3395.

[4] Maoz R. and Sagiv J., J. Colloid Interface Sci. 100 (1984) 465-496.

[5] Wasserman S. R., Tao Y.-T., and Whitesides G. M., Langmuir 5 (1989) 1074-1087.

[6] Mustre de Leon J., Rehr J. J., Zabinsky S. I., and Albers R. C., Phys. Rev. B44 (1991) 4146-4156.

[7] Lytle F. W., Greegor R. B., and Panson A. J., Phys. Rev. B37 (1988) 1550-1562.

[8] Smith T. A., Penner-Hahn J. E., Berding M. A., Doniach S., and Hodgson K. O., J. Am. Chem. Soc. 107 (1985) 5945-5955.

[9] Wasserman S. R., Anderson K. B., Song K., Yuchs S. E., and Marshall C. L., US Patent Applied For.

[10] Hench L. L., Clark D. E., and Yen-Bower E. L., Nucl. Chem. Waste Manag. 1 (1980) 59-75. 


\section{Smectite Clay}

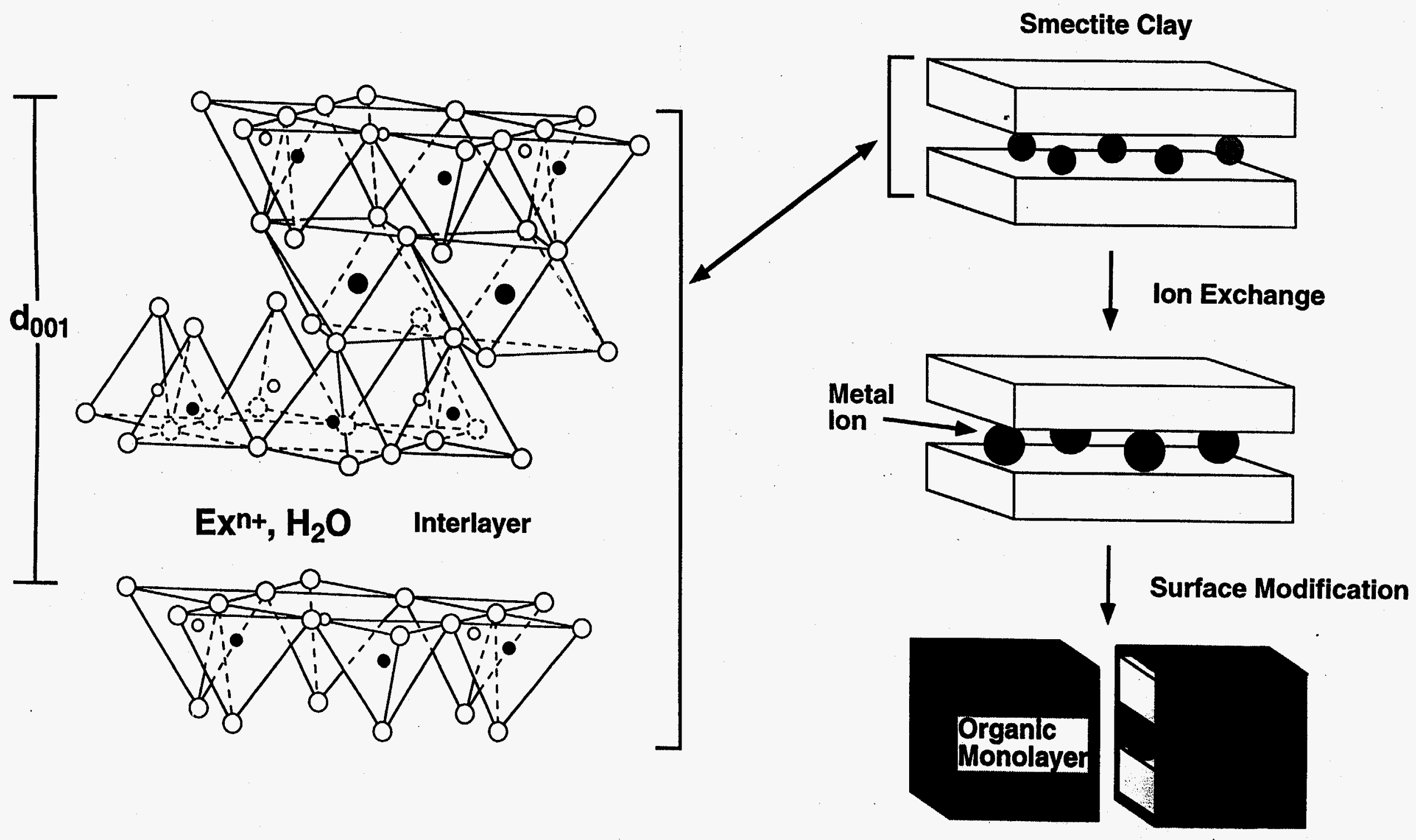



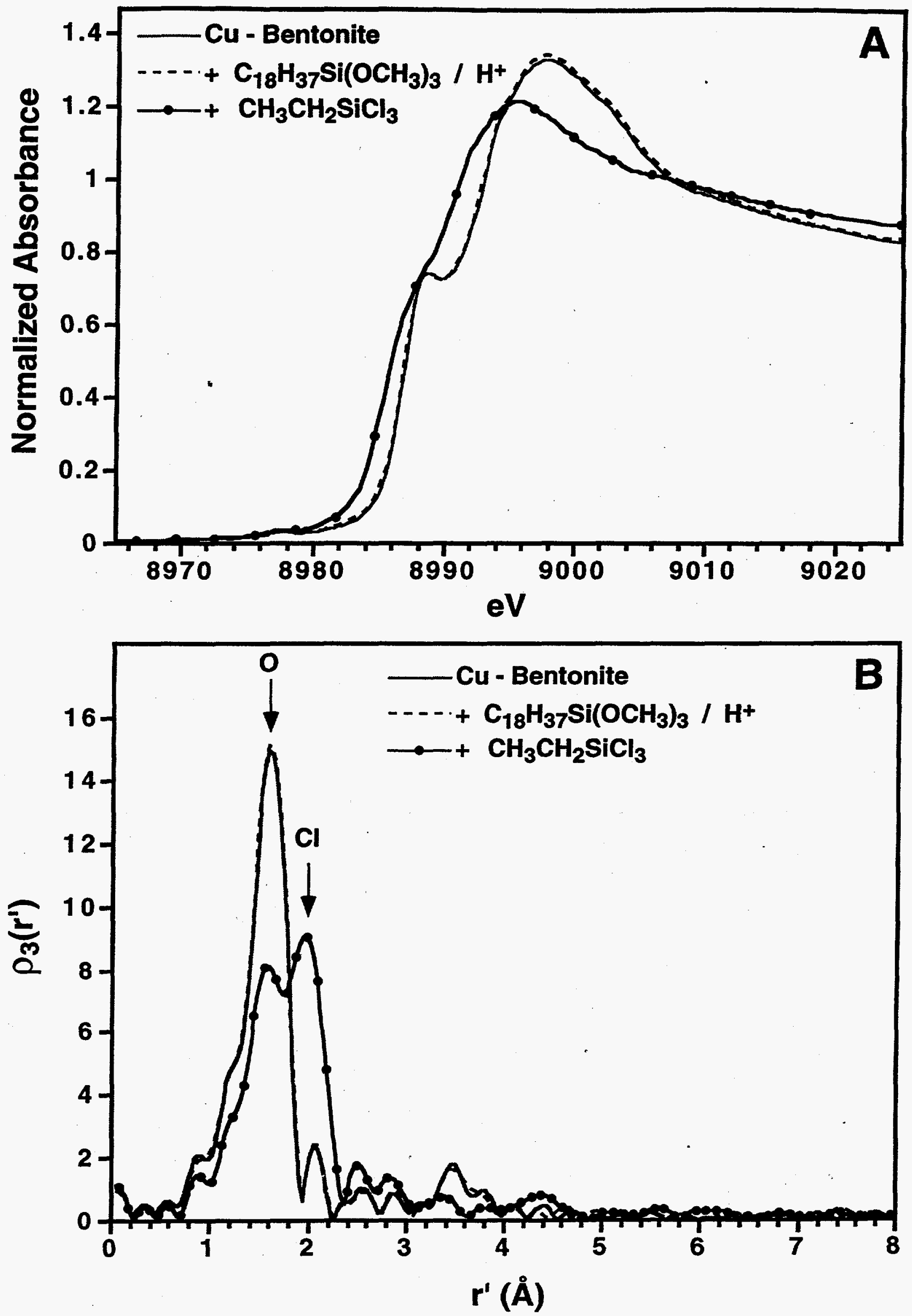

Figure 2 

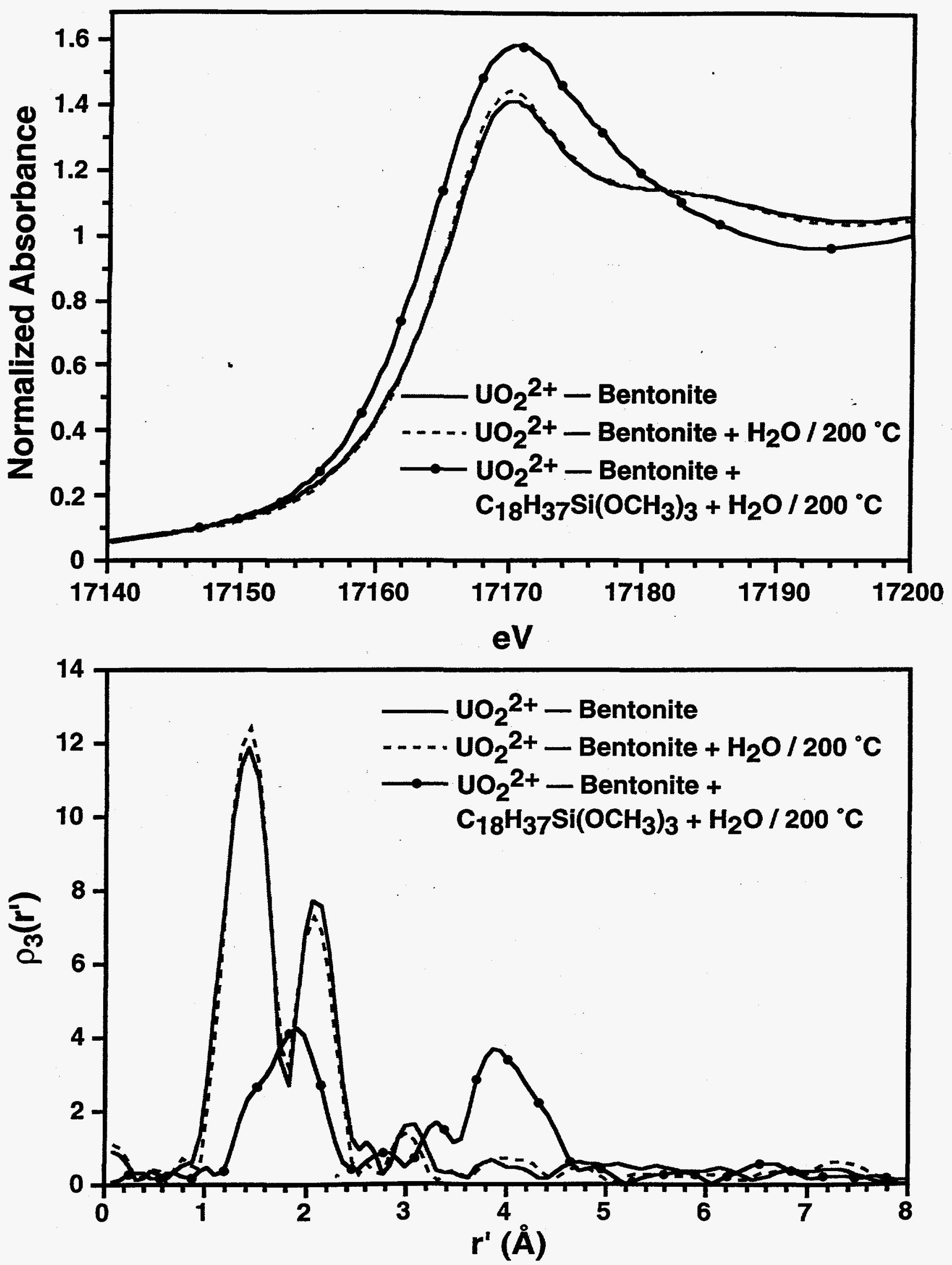